\title{
CONVERGENCE RATES OF MONOTONE SCHEMES FOR CONSERVATION LAWS WITH DISCONTINUOUS FLUX*
}

\author{
JAYESH BADWAIK ${ }^{\dagger}$ AND ADRIAN M. RUF
}

\begin{abstract}
We prove that a class of monotone finite volume schemes for scalar conservation laws with discontinuous flux converge at a rate of $\sqrt{\Delta x}$ in $\mathrm{L}^{1}$, whenever the flux is strictly monotone in $u$ and the spatial dependency of the flux is piecewise constant with finitely many discontinuities. We also present numerical experiments to illustrate the main result. To the best of our knowledge, this is the first proof of any type of convergence rate for numerical methods for conservation laws with discontinuous, nonlinear flux.

Our proof relies on convergence rates for conservation laws with initial and boundary value data. Since those are not readily available in the literature we establish convergence rates in that case en passant in the Appendix.
\end{abstract}

Key words. hyperbolic conservation laws, discontinuous flux, numerical methods, convergence rate

AMS subject classifications. 35L65, 65M08, 65M12, 35R05

1. Introduction. We prove a convergence rate for a class of monotone, upwindtype finite volume schemes for scalar conservation laws with discontinuous flux of the form

$$
\begin{array}{cl}
u_{t}+f(k(x), u)_{x}=0, & (x, t) \in \mathbb{R} \times(0, T), \\
u(x, 0)=u_{0}(x), \quad x \in \mathbb{R} .
\end{array}
$$

Here, we assume that the flux $f$ is strictly monotone in $u$ and has a discontinuous spatial dependency through the coefficient $k$ which is piecewise constant with finitely many discontinuities.

Main Theorem. Let $f$ be strictly monotone in $u$ in the sense that $f_{u}>0, k$ piecewise constant with finitely many discontinuities, and $u_{0} \in\left(\mathrm{L}^{1} \cap \mathrm{BV}\right)(\mathbb{R})$. Then all monotone finite volume methods with the upwind property which obey the discrete Rankine-Hugoniot condition across the discontinuities of $k$ converge at a rate of $\sqrt{\Delta x}$ to the unique entropy solution of the conservation law (1.1).

The full theorem is stated in Section 5. Our proof uses the Rankine-Hugoniot condition at the discontinuities of $k$ to break down the problem into finitely many initialboundary value problems for each of which we will prove a convergence rate using the classical 'doubling of variables' technique.

1.1. Background on conservation laws with discontinuous fluxes. Problem (1.1) is of great practical interest in several areas of physics and engineering. In particular, it arises in modeling traffic flow on highways with changing road conditions (see [25]), in the modeling of two-phase flow in a porous medium (see [13, 32]), and in modeling sedimentation processes (see $[11,7]$ ).

\footnotetext{
*Submitted August 23, 2019.

Funding: Both authors have received funding from the European Unions Framework Programme for Research and Innovation Horizon 2020 (2014-2020) under the Marie Skłodowska-Curie Grant Agreement No. 642768. In addition, J. Badwaik was supported by the Priority Programme 1648 of the German Science Foundation and A. M. Ruf has received funding from NFRDAAD grant No. 281268.

${ }^{\dagger}$ Department of Mathematics, University of Würzburg, Germany (badwaik.jayesh@gmail.com).

‡Seminar for Applied Mathematics, ETH Zürich, Switzerland (adrian.ruf@sam.math.ethz.ch).
} 
The flux in (1.1) depends on the space variable through a coefficient $k$ which may be discontinuous. The dependence can for example be of the additive type, i.e., $f(k(x), u)=f(u)-k(x)$ (see [14]), or of the multiplicative type, i.e., $f(k(x), u)=$ $k(x) f(u)$ which is more common (see e.g. [37]). However, for the sake of generality we do not assume any particular algebraic structure of the flux $f(k(x), u)$ here. The case we consider in this paper where $k$ is piecewise constant with finitely many discontinuities corresponds to switching from one $u$-dependent flux function, $f^{(i-1)}$, to another, $f^{(i)}$, across a discontinuity $\xi_{i}$ of $k$. When $k$ has just one discontinuity - the so-called 'two flux' case - given by

$$
u_{t}+(H(x) f(u)+(1-H(x)) g(u))_{x}=0
$$

where $H$ is the Heaviside function was studied in a series of papers by Adimurthi, Mishra, and Gowda (see [27, 2, 1] and references therein). Most notably, in [2], the authors showed existence of infinitely many $\mathrm{L}^{1}$-stable semi-groups of solutions to (1.2). We remark that, because of the assumption that $k$ is piecewise constant, the convergence rate of monotone schemes for (1.2) will be the building block for the general case of (1.1).

Equations of type (1.1) have been dealt with extensively in the literature from a purely academic point of view as well as with a specific application in mind. In $[12,13]$, Gimse and Risebro calculated solutions for the Riemann problem assuming convexity of the flux in $u$ and used the solutions to show existence of a weak solution for the general Cauchy problem with a front tracking algorithm. Other results based on the front tracking algorithm were obtained in [20], [19], [6], [21], [7], and in [9] with a time-dependent discontinuous coefficient. Out of the aforementioned results, we want to highlight [6] from Baiti and Jensen who proved existence and uniqueness of entropy solutions in the case that the flux is strictly monotone in $u$ which is the case we consider in this paper as well.

The first results for finite volume schemes for (1.1) (assuming a multiplicative spatial dependency) were obtained by Towers in [37, 38]. Specifically, in [37], the author developed staggered versions of the Godunov and Engquist-Osher schemes for the case where $f$ is convex in $u$ and $k$ is strictly positive. In [38] similar results were proved for the case of non-convex fluxes. In [16], Karlsen, Risebro, and Towers studied (1.1) with an added degenerate parabolic term using an Engquist-Osher-type scheme and in [17] the authors proved existence of the vanishing viscosity limit using compensated compactness. In [18], Karlsen and Towers showed convergence of the Lax-Friedrichs scheme for (1.1) (with a time-dependent discontinuous coefficient). They were able to handle very general fluxes and sign-changing coefficients by using compensated compactness.

A general framework for well-posedness of (1.2) was proposed by Andreianov, Karlsen, and Risebro in [3].

Lastly, we want to point out that the monotonicity assumption, $f_{u}>0$ we use in this paper implies that the equivalent system

$$
\begin{array}{r}
u_{t}+f(k, u)_{x}=0, \\
k_{t}=0
\end{array}
$$

is hyperbolic and not resonant, see [37, 38, 19, 20, 21].

1.2. Background on convergence rates. When dealing with numerical methods for (1.1), where an approximate solution $u_{\Delta t}$ depends on a grid discretization 
parameter $\Delta x$, having a provable bound of the type

$$
\left\|u(T)-u_{\Delta t}(T)\right\|_{L^{1}(\mathbb{R})} \leq C \Delta x^{r},
$$

- specifying how fast the numerical scheme converges - is highly desirable. Specifically, convergence rates can be used for a posteriori error based mesh adaptation [39] and optimal design of multilevel Monte Carlo methods [5]. To this date the only result concerning convergence rates of finite volume schemes for conservation laws with discontinuous flux is due to Wen and Jin and pertains the most basic case of the linear advection equation with piecewise constant wave speed that changes across a single discontinuity, [40]. So far, in the nonlinear case, convergence rates of finite volume schemes for (1.1) are only available in the absence of a spatial dependency, i.e., $k$ being constant. The main difficulty in obtaining convergence rates when the flux has a discontinuous spatial dependency is that in this case the classical 'doubling of variables' technique (see [22]) involves both, terms with $k(x)$ and terms with $k(y)$.

In the case of a spatially independent flux the seminal paper by Kuznetsov [23] shows that monotone schemes converge towards the entropy solution of (1.1) without spatial dependency at a rate of $\mathcal{O}(\sqrt{\Delta x})$ in $\mathrm{L}^{1}$. This rate was proved for initial data in $\left(\mathrm{L}^{1} \cup \mathrm{BV}\right)(\mathbb{R})$, and in this generality the rate $\mathcal{O}(\sqrt{\Delta x})$ is in fact optimal, as was shown by Şabac in [34]. There are certain classes of initial data for which higher orders of convergence for monotone schemes have been shown, e.g. Teng and Zhang [36] showed a convergence rate of $\mathcal{O}(\Delta x)$ for the case of piecewise constant initial data. See also [33] for a more comprehensive overview of convergence rate results for (1.1) without spatial dependency.

An alternative approach to convergence rates in the case where the flux only depends on $u$ was initiated by Nessyahu, Tadmor, and Tassa [28, 29]. The authors used the Wasserstein distance instead of the $\mathrm{L}^{1}$ norm and were able to show that a large class of monotone schemes converge at a rate of $\mathcal{O}(\Delta x)$ in the Wasserstein distance for $\mathrm{Lip}^{+}$-bounded, compactly supported initial data. This rate was recently proved to be optimal by Ruf, Sande, and Solem [33].

Since the proof of our main theorem makes use of convergence rates for conservation laws on bounded domains, it is worth mentioning that Ohlberger and Vovelle claimed a convergence rate of $\mathcal{O}\left(\Delta x^{1 / 3}\right)$ for conservation laws with initial and boundary data in one dimension [30, p. 135]. In our specific case of a strictly monotone flux however, we are able to prove a better rate of $\mathcal{O}(\sqrt{\Delta x})$.

1.3. Outline of the paper. We have organized the paper in the following way. In Section 2, we will define entropy solutions of (1.1) and show that - when restricted to a subdomain between two neighboring discontinuities of $k$ - they are entropy solutions of a certain initial boundary value problem with spatially independent flux. Here the respective boundary datum is given through the Rankine-Hugoniot condition across a discontinuity of $k$. In Section 3, we describe our finite volume scheme and show that we can establish a convergence rate of our numerical method for (1.1) by proving a convergence rate for each of those initial-boundary value problems. In Section 4, we start by considering just one discontinuity of $k$, i.e., Equation (1.2), and proving a convergence rate on $\mathbb{R}^{-}$and $\mathbb{R}^{+}$separately. Section 5 contains the statement and proof of the main result where we use the translation invariance of conservation laws and the results of the previous section in our main proof. Section 6 describes numerical experiments that illustrate our convergence rate result as well as the class of fluxes that is covered by our theory. In Section 7, we summarize the findings of this paper and provide an outlook. Lastly, in Appendix A, we show that - 
with minimal changes - our results can be applied to general initial-boundary value problems where the prescribed boundary datum is arbitrary.

2. Preliminaries. Throughout this paper, we will assume that the initial datum $u_{0}$ is integrable, bounded, and of finite total variation, i.e., $u_{0} \in\left(\mathrm{L}^{1} \cap \mathrm{BV}\right)(\mathbb{R})$, and that $f$ is strictly monotone in $u$, i.e., $f_{u} \geq \alpha>0$. Further, we will denote the discontinuities of $k$ as $\xi_{1}, \ldots, \xi_{N}$ and the interval between two adjacent discontinuities as $D_{i}=\left(\xi_{i}, \xi_{i+1}\right), i=0, \ldots, N$. Here, we used the notation $\xi_{0}=-\infty$ and $\xi_{N+1}=\infty$. Then we can write

$$
f(k(x), \cdot)=: f^{(i)}(\cdot) \quad \text { for } x \in D_{i} .
$$

We will consider entropy solutions of Equation (1.1) in the following sense.

Definition 2.1 (Entropy solution). We say $u \in \mathcal{C}\left([0, T] ; \mathrm{L}^{1}(\mathbb{R})\right) \cap \mathrm{L}^{\infty}((0, T) \times \mathbb{R})$ is an entropy solution of Equation (1.1) if for all $c \in \mathbb{R}$

$$
\begin{aligned}
\sum_{i=0}^{N}\left(\int_{0}^{T} \int_{D_{i}}\left(\left|u-c_{i}\right| \varphi_{t}+\operatorname{sign}\left(u-c_{i}\right)\left(f^{(i)}(u)-f^{(i)}\left(c_{i}\right)\right) \varphi_{x}\right) \mathrm{d} x \mathrm{~d} t\right. \\
\left.\quad-\int_{D_{i}}\left|u(x, T)-c_{i}\right| \varphi(x, T) \mathrm{d} x+\int_{D_{i}}\left|u_{0}(x)-c_{i}\right| \varphi(x, 0)\right) \mathrm{d} x \\
-\int_{0}^{T} \operatorname{sign}\left(u\left(\xi_{i+1}-, t\right)-c_{i}\right)\left(f^{(i)}\left(u\left(\xi_{i+1}-, t\right)\right)-f^{(i)}\left(c_{i}\right)\right) \varphi\left(\xi_{i+1}, t\right) \mathrm{d} t \\
\left.\quad+\int_{0}^{T} \operatorname{sign}\left(u\left(\xi_{i}+, t\right)-c_{i}\right)\left(f^{(i)}\left(u\left(\xi_{i}+, t\right)\right)-f^{(i)}\left(c_{i}\right)\right) \varphi\left(\xi_{i}, t\right) \mathrm{d} t\right) \geq 0 .
\end{aligned}
$$

for all nonnegative $\varphi \in \mathcal{C}^{\infty}(\mathbb{R} \times[0, T])$. Here, the $c_{i}$ are given by $c_{0}:=c$ and

$$
c_{i+1}=\left(f^{(i+1)}\right)^{-1}\left(f^{(i)}\left(c_{i}\right)\right) \quad \text { for } i=1, \ldots, N .
$$

Remark 2.2. Note that, due to the monotonicity of the fluxes $f^{(i)}$, the inverse of $f^{(i)}$ used in (2.1) and throughout this paper exists.

Remark 2.3. Note that existence and uniqueness of entropy solutions of Equation (1.1) are guaranteed by the theory developed by Baiti and Jensen in [6] using adapted entropies in the sense above (cf. also [4] where adapted entropies are used as well). In particular, the traces in Defintion 2.1 are well defined (cf. [3, Remark 2.3])

Remark 2.4. Like for conservation laws without (discontinuous) spatial dependency of the flux, a Rankine-Hugoniot-type argument shows that weak solutions of (1.1) necessarily satisfy the Rankine-Hugoniot condition across all discontinuities $\xi_{i}$, i.e.,

$$
f^{(i-1)}\left(u\left(\xi_{i}-, t\right)\right)=f^{(i)}\left(u\left(\xi_{i}+, t\right)\right) .
$$

The following observation is at the heart of the proof of the main result. The entropy solution $u$ of (1.1) can be decomposed as $u=\sum_{i=0}^{N} u^{(i)}$ where $u^{(i)}:=u \mathbb{1}_{D_{i} \times[0, T]}$ such that $u^{(0)}$ solves

$$
\begin{array}{cl}
u_{t}^{(0)}+f^{(0)}\left(u^{(0)}\right)_{x}=0, & (x, t) \in D_{0} \times(0, T), \\
u^{(0)}(x, 0)=u_{0}(x), & x \in D_{0}
\end{array}
$$


and $u^{(i)}$ solves

$$
\begin{aligned}
u_{t}^{(i)}+f^{(i)}\left(u^{(i)}\right)_{x}=0, & (x, t) \in D_{i} \times(0, T), \\
u^{(i)}(x, 0)=u_{0}(x), & x \in D_{i}, \\
u^{(i)}\left(\xi_{i}+, t\right)=\left(f^{(i)}\right)^{-1}\left(f^{(i-1)}\left(u^{(i-1)}\left(\xi_{i}-, t\right)\right)\right), & t \in(0, T)
\end{aligned}
$$

for $i=1, \ldots, N$ (cf. Definitions 4.1 and 4.5 below). Note that the boundary condition on the domain $D_{i}, i=1, \ldots, N$, given by the last line of (2.4) reflects the RankineHugoniot condition (2.2).

Conversely, if $u^{(0)}$ is the entropy solution of (2.3) on $D_{0}$ and $u^{(i)}$ is the entropy solution of (2.4) on $D_{i}$ for $i=1, \ldots, N$, then the composite function $u:=\sum_{i=0}^{N} u^{(i)}$ is the entropy solution of (1.1) in the sense of Definition 2.1. This can be seen by adding the entropy inequalities of $u^{(i)}$ and choosing the respective constant in each entropy inequality in accordance with (2.1).

In the remainder of the paper, we will construct a numerical scheme which satisfies the Rankine-Hugoniot condition (2.2) (or, equivalently, the last line of (2.4)) across the discontinuities of $k$ on the discrete level. This scheme, when restricted to the subdomain $D_{i}$, will converge towards the entropy solution on $D_{i}$. The discrete Rankine-Hugoniot condition will then allow us to break down the problem of finding a convergence rate on the whole real line to finding convergence rates on each of the subdomains $D_{i}$.

3. The numerical scheme. We discretize the domain $\mathbb{R} \times[0, T]$ using the spatial and temporal grid discretization parameters $\Delta x$ and $\Delta t$. The resulting grid cells then are $\mathcal{C}_{j}=\left(x_{j-1 / 2}, x_{j+1 / 2}\right)$ and $\mathcal{C}^{n}=\left(t^{n}, t^{n+1}\right)$ for points $x_{j+1 / 2}$, such that $x_{j+1 / 2}-$ $x_{j-1 / 2}=\Delta x, j \in \mathbb{Z}$, and $t^{n}=n \Delta t$ for $n=0, \ldots, M+1$. Note that $T=(M+1) \Delta t$. Further we write $\mathcal{C}_{j}^{n}$ to denote the rectangle $\mathcal{C}_{j} \times \mathcal{C}^{n}$.

In the following we will assume that the grid is aligned in such a way that all discontinuities of $k$ lie on cell interfaces, i.e., $\xi_{i}=x_{P_{i}-1 / 2}$ for some integers $P_{i}, i=$ $1, \ldots, N$. In general this can easily be achieved by considering a globally non-uniform grid that is uniform on each $D_{i}$ and then taking $\Delta x=\max _{i=0, \ldots, N} \Delta x_{i}$ where $\Delta x_{i}$ is the grid discretization parameter in $D_{i}$. For simplicity however, here we will assume that the grid is uniform on the whole real line.

Further, we will consider two-point numerical fluxes $F(u, v)$ that have the upwind property such that if $f^{\prime} \geq 0$ then $F(u, v)=f(v)$. Such fluxes include the upwind flux, the Godunov flux, and the Engquist-Osher flux. Thus, the numerical scheme we will analyze is the following:

$$
\begin{array}{cl}
u_{j}^{n+1}=u_{j}^{n}-\lambda\left(f^{(i)}\left(u_{j}^{n}\right)-f^{(i)}\left(u_{j-1}^{n}\right)\right), & n \geq 0, P_{i}<j<P_{i+1}, 0 \leq i \leq N \\
u_{j}^{0}=\frac{1}{\Delta x} \int_{\mathcal{C}_{j}} u_{0}(x) \mathrm{d} x, \quad j \in \mathbb{Z}, & \\
u_{P_{i}}^{n+1}=\left(f^{(i)}\right)^{-1}\left(f^{(i-1)}\left(u_{P_{i}-1}^{n+1}\right)\right), & n \geq 0,0<i \leq N
\end{array}
$$

where $\lambda=\Delta t / \Delta x$. We assume that the grid discretization parameters satisfy the CFL condition

$$
\max _{i} \max _{u}\left(f^{(i)}\right)^{\prime}(u) \lambda \leq 1 .
$$

Note that the last line of (3.1) represents a discrete version of the RankineHugoniot condition (2.2). Here, we use the ghost cells $\mathcal{C}_{P_{i}}, i=1, \ldots, N$ to explicitely 
enforce the Rankine-Hugoniot condition on the discrete level. While this makes the numerical scheme (3.1) non-conservative, the convergence result in this paper, coupled with the fact that the limit is conservative, shows that the contribution of the nonconservative part of the scheme vanishes in the limit.

To get a convergence rate of the numerical scheme (3.1) we decompose the entropy solution $u$ as $u=\sum_{i=0}^{N} u^{(i)}$ where $u^{(i)}, i=0, \ldots, N$, are the respective entropy solutions on $D_{i}$ and the numerical solution $u_{\Delta t}$ as $\sum_{i=0}^{N} u_{\Delta t}^{(i)}$ where

$$
u_{\Delta t}^{(i)}(x, t)= \begin{cases}u_{j}^{n} & \text { if }(x, t) \in \mathcal{C}_{j}^{n} \subset D_{i} \times \mathcal{C}^{n} \\ 0 & \text { otherwise }\end{cases}
$$

Then we have

$$
\left\|u(T)-u_{\Delta t}(T)\right\|_{\mathrm{L}^{1}(\mathbb{R})}=\sum_{i=0}^{N}\left\|u^{(i)}(T)-u_{\Delta t}^{(i)}(T)\right\|_{\mathrm{L}^{1}\left(D_{i}\right)}
$$

and the problem of finding a convergence rate for $u_{\Delta t}$ can be broken down to finding convergence rates for $u_{\Delta t}^{(i)}$ on each of the subdomains $D_{i}$. In the following sections, we will show that

$$
\left\|u^{(i)}(T)-u_{\Delta t}^{(i)}(T)\right\|_{L^{1}\left(D_{i}\right)} \leq C \sqrt{\Delta x}
$$

Note that convergence of the numerical scheme (3.1) towards the entropy solution of (1.1) follows from our convergence rate estimate. At this point we want to point out that instead of assuming $f_{u}>0$ our proof can readily be adapted for the case $f_{u}<0$.

4. Convergence rates for fluxes with one discontinuity. We will first consider the case where $k$ has just two constant values separated by a discontinuity $\xi_{1}$ and for ease of notation we will assume that $\xi_{1}=0$. Further, we will denote the flux left of $\xi_{1}$ as $g$ and right of $\xi_{1}$ as $f$. In order to get a convergence rate for problem (1.1) we will derive convergence rates on $D_{0}=\mathbb{R}^{-}$, on $D_{1}=\mathbb{R}^{+}$, and on $(0, L)$ for $L>0$.

4.1. Convergence rate estimates on $\mathbb{R}^{-}$. As a first step we consider the initial value problem

$$
\begin{array}{ll}
u_{t}+g(u)_{x}=0, & (x, t) \in \mathbb{R}^{-} \times(0, T), \\
u(x, 0)=u_{0}(x), & x \in \mathbb{R}^{-}
\end{array}
$$

on $\mathbb{R}^{-}$with the flux $g$ being strictly monotone and consider entropy solutions in the following sense.

Definition 4.1 (Entropy solution on $\left.\mathbb{R}^{-}\right)$. We say $u \in \mathcal{C}\left([0, T] ; \mathrm{L}^{1}\left(\mathbb{R}^{-}\right)\right) \cap$ $\mathrm{L}^{\infty}\left((0, T) \times \mathbb{R}^{-}\right)$is an entropy solution of Equation (4.1) if for all $c \in \mathbb{R}$,

$$
\begin{aligned}
\int_{0}^{T} \int_{\mathbb{R}^{-}}\left(|u-c| \varphi_{t}+|g(u)-g(c)| \varphi_{x}\right) \mathrm{d} x \mathrm{~d} t-\int_{\mathbb{R}^{-}}|u(x, T)-c| \varphi(x, T) \mathrm{d} x \\
\left.\quad+\int_{\mathbb{R}^{-}}\left|u_{0}(x)-c\right| \varphi(x, 0)\right) \mathrm{d} x-\int_{0}^{T}|g(u(0-, t))-g(c)| \varphi(0, t) \mathrm{d} t \geq 0
\end{aligned}
$$

for all nonnegative $\varphi \in \mathcal{C}^{\infty}((-\infty, 0] \times[0, T])$. 
Note that here $u(0-, t)$ denotes the limit of $u(x, t)$ as $x \rightarrow 0$ from the left.

As before, we will write $\mathcal{C}_{j}=\left(x_{j-1 / 2}, x_{j+1 / 2}\right), j \in \mathbb{Z}$, where now $x_{-1 / 2}:=0$. Our numerical scheme then reads

$$
\begin{gathered}
u_{j}^{n+1}=u_{j}^{n}-\lambda\left(g\left(u_{j}^{n}\right)-g\left(u_{j-1}^{n}\right)\right), \quad j<0, n \geq 0, \\
u_{j}^{0}=\frac{1}{\Delta x} \int_{\mathcal{C}_{j}} u_{0}(x) \mathrm{d} x, \quad j<0
\end{gathered}
$$

where $\lambda=\Delta t / \Delta x$ satisfies the CFL condition (3.2).

We note that the numerical scheme satisfies a discrete entropy inequality away from the spatial boundary

$$
D_{+}^{t} \eta_{j}^{n}+D_{-} q_{j}^{n} \leq 0, \quad n \geq 1, j<0
$$

which can be seen by adopting the classical Crandall-Majda arguments in [10, Prop. 4.1] for $j<0$. Here, $\eta_{j}^{n}=\eta\left(u_{j}^{n}, c\right)=\left|u_{j}^{n}-c\right|, q_{j}^{n}=q\left(u_{j}^{n}, c\right)=\operatorname{sign}\left(u_{j}^{n}-c\right)\left(g\left(u_{j}^{n}\right)-\right.$ $g(c))=\left|g\left(u_{j}^{n}\right)-g(c)\right|$ and

$$
D_{+}^{t} a^{n}=\frac{a^{n+1}-a^{n}}{\Delta t} \quad \text { and } \quad D_{-} a_{j}=\frac{a_{j}-a_{j-1}}{\Delta x}
$$

denote standard difference operators.

In order to derive convergence rates we will develop a Kuznetsov-type lemma in the following. For any function $u \in \mathcal{C}\left([0, T] ; \mathrm{L}^{1}\left(\mathbb{R}^{-}\right)\right)$we define

$$
\begin{aligned}
L(u, c, \varphi)= & \int_{0}^{T} \int_{\mathbb{R}^{-}}\left(|u-c| \varphi_{t}+q(u, c) \varphi_{x}\right) \mathrm{d} x \mathrm{~d} t-\int_{\mathbb{R}^{-}}|u(x, T)-c| \varphi(x, T) \mathrm{d} x \\
& +\int_{\mathbb{R}^{-}}\left|u_{0}(x)-c\right| \varphi(x, 0) \mathrm{d} x-\int_{0}^{T} q(u(0-, t), c) \varphi(0, t) \mathrm{d} t
\end{aligned}
$$

where $q(u, c)=|g(u)-g(c)|$ is the Kružkov entropy flux. Note that if $u$ is an entropy solution of (4.1) then $L(u, c, \varphi) \geq 0$ for all $c \in \mathbb{R}$ and test functions $\varphi \geq 0$. We now take $c=v(y, s)$ and the test function

$$
\varphi(x, t, y, s)=\omega_{\varepsilon}(x-y) \omega_{\varepsilon_{0}}(t-s)
$$

where $\omega_{\varepsilon}, \omega_{\varepsilon_{0}}$ are standard symmetric mollifiers for $\varepsilon, \varepsilon_{0}>0$. Note that $\varphi_{t}=-\varphi_{s}$, $\varphi_{x}=-\varphi_{y}$ and

$$
\varphi(x, t, y, s)=\varphi(y, t, x, s)=\varphi(y, s, x, t)=\varphi(x, s, y, t)
$$

as well as

$$
\begin{array}{ll}
\int_{\mathbb{R}} \omega_{\varepsilon}(x-y) \mathrm{d} y \leq 1, & \int_{\mathbb{R}}\left|\omega_{\varepsilon}^{\prime}(x-y)\right| \mathrm{d} y \leq \frac{C}{\varepsilon}, \\
\int_{0}^{T} \omega_{\varepsilon_{0}}(t-s) \mathrm{d} s \leq 1, & \int_{0}^{T}\left|\omega_{\varepsilon_{0}}^{\prime}(t-s)\right| \mathrm{d} s \leq \frac{C}{\varepsilon_{0}}
\end{array}
$$

for all $x \in \mathbb{R}, t \in[0, T]$. Let now

$$
\Lambda_{\varepsilon, \varepsilon_{0}}(u, v)=\int_{0}^{T} \int_{\mathbb{R}^{-}} L(u, v(y, s), \varphi(\cdot, \cdot, y, s)) \mathrm{d} y \mathrm{~d} s .
$$


For functions $w \in \mathcal{C}\left([0, T] ; \mathrm{L}^{1}\left(\mathbb{R}^{-}\right)\right)$, we further define the moduli of continuity

$$
\begin{aligned}
\nu_{t}\left(w, \varepsilon_{0}\right) & =\sup _{|\sigma| \leq \varepsilon_{0}}\|w(\cdot, t+\sigma)-w(\cdot, t)\|_{\mathrm{L}^{1}\left(\mathbb{R}^{-}\right)}, \\
\mu(w(\cdot, t), \varepsilon) & =\sup _{|z| \leq \varepsilon}\|w(\cdot+z, t)-w(\cdot, t)\|_{\mathrm{L}^{1}\left(\mathbb{R}^{-}\right)} .
\end{aligned}
$$

Lemma 4.2 (Kuznetsov-type lemma). Let $u$ be the entropy solution of (4.1). Then, for any function $v:[0, T] \rightarrow\left(\mathrm{L}^{1} \cap \mathrm{BV}\right)\left(\mathbb{R}^{-}\right)$such that the one-sided limits $v(t \pm)$ exist in $\mathrm{L}^{1}$, we have

$$
\begin{aligned}
& \begin{array}{l}
\|u(\cdot, T)-v(\cdot, T)\|_{\mathrm{L}^{1}\left(\mathbb{R}^{-}\right)} \\
\quad+\int_{0}^{T} \int_{\mathbb{R}^{-}} \int_{0}^{T}(q(u(0-, t), v(y, s))+q(v(0-, t), u(y, s))) \varphi(0, t, y, s) \mathrm{d} t \mathrm{~d} y \mathrm{~d} s \\
\leq\left\|u_{0}-v(\cdot, 0)\right\|_{\mathrm{L}^{1}\left(\mathbb{R}^{-}\right)}-\Lambda_{\varepsilon, \varepsilon_{0}}(v, u) \\
\left.\left.\quad+C\left(\varepsilon+\varepsilon_{0}+\nu_{T}\left(v, \varepsilon_{0}\right)+\nu_{0}\left(v, \varepsilon_{0}\right)+\mu(v(\cdot, T), \varepsilon)\right)+\mu(v(\cdot, 0), \varepsilon)\right)\right)
\end{array} \quad
\end{aligned}
$$

for some constant $C$ independent of $\varepsilon$ and $\varepsilon_{0}$.

Proof. Using that $\varphi_{t}=-\varphi_{s}, \varphi_{x}=-\varphi_{y}$ and the symmetry relations (4.4) we get

$$
\begin{aligned}
\Lambda_{\varepsilon, \varepsilon_{0}}(u, v) & -\Lambda_{\varepsilon, \varepsilon_{0}}(v, u) \\
& -\underbrace{\int_{0}^{T} \int_{\mathbb{R}^{-}} \int_{\mathbb{R}^{-}}(|u(x, T)-v(y, s)|+|v(x, T)-u(y, s)|) \varphi(x, T, y, s) \mathrm{d} x \mathrm{~d} y \mathrm{~d} s}_{=: \mathbf{A}} \\
& +\underbrace{\int_{0}^{T} \int_{\mathbb{R}^{-}} \int_{\mathbb{R}^{-}}\left(\left|u_{0}(x, t)-v(y, s)\right|+|v(x, 0)-u(y, s)|\right) \varphi(x, 0, y, s) \mathrm{d} x \mathrm{~d} y \mathrm{~d} s}_{=: \mathbf{B}} \\
& -\underbrace{\int_{0}^{T} \int_{\mathbb{R}^{-}} \int_{0}^{T}(q(u(0-, t), v(y, s))+q(v(0-, t), u(y, s))) \varphi(0, t, y, s) \mathrm{d} t \mathrm{~d} y \mathrm{~d} s}_{=: \mathbf{C}} .
\end{aligned}
$$

Since $u$ is an entropy solution we find

$$
0 \leq \Lambda_{\varepsilon, \varepsilon_{0}}(u, v)=-\Lambda_{\varepsilon, \varepsilon_{0}}(v, u)-\mathbf{A}+\mathbf{B}-\mathbf{C}
$$

and thus

$$
\mathbf{A}+\mathbf{C} \leq-\Lambda_{\varepsilon, \varepsilon_{0}}(v, u)+\mathbf{B}
$$

The terms $\mathbf{A}$ and $\mathbf{B}$ also appear in the case of an unbounded spatial domain and can be estimated by

$$
\begin{aligned}
\mathbf{A} \geq & \|u(\cdot, T)-v(\cdot, T)\|_{\mathrm{L}^{1}\left(\mathbb{R}^{-}\right)} \\
& -\frac{1}{2}\left(\nu_{T}\left(u, \varepsilon_{0}\right)+\mu(u(\cdot, T), \varepsilon)+\nu_{T}\left(v, \varepsilon_{0}\right)+\mu(v(\cdot, T), \varepsilon)\right)
\end{aligned}
$$


and

$$
\mathbf{B} \leq\left\|u_{0}-v(\cdot, 0)\right\|_{\mathrm{L}^{1}\left(\mathbb{R}^{-}\right)}+\frac{1}{2}\left(\nu_{0}\left(u, \varepsilon_{0}\right)+\mu\left(u_{0}, \varepsilon\right)+\nu_{0}\left(v, \varepsilon_{0}\right)+\mu(v(\cdot, 0), \varepsilon)\right),
$$

see [8] or [15] for details. Lastly, due to the Lipschitz continuity in time and the TVD property (see [15, Thm. 2.15] and [15, Lem. A.1]) the entropy solution of (4.1) satisfies

$$
\begin{aligned}
\nu_{0}\left(u, \varepsilon_{0}\right), \nu_{T}\left(u, \varepsilon_{0}\right) & \leq C \operatorname{TV}\left(u_{0}\right) \varepsilon_{0} \\
\text { and } \quad \mu\left(u_{0}, \varepsilon\right), \mu(u(\cdot, T), \varepsilon) & \leq \operatorname{TV}\left(u_{0}\right) \varepsilon
\end{aligned}
$$

which completes the proof.

In order to derive a convergence rate the next step is to estimate the term $\Lambda_{\varepsilon, \varepsilon_{0}}\left(u_{\Delta t}, u\right)$.

LEMMA 4.3. The estimate

$$
-\Lambda_{\varepsilon, \varepsilon_{0}}\left(u_{\Delta t}, u\right) \leq C\left(\Delta x+\frac{\Delta x}{\varepsilon}+\frac{\Delta t}{\varepsilon_{0}}\right)
$$

holds for some constant $C$ independent of $\Delta x, \Delta t, \varepsilon$, and $\varepsilon_{0}$.

Proof. The proof of Lemma 4.3 for conservation laws on the real line can be found e.g. in [15]. Here, we only need to replace any sum of the form $\sum_{j=-\infty}^{\infty}$ by $\sum_{-\infty}^{-1}$ and note that the boundary term in space cancels after integration by parts. Confer also the proof of Lemma 4.9 in Section 4.2 for details.

TheOREM 4.4 (Convergence rate on $\mathbb{R}^{-}$). Let $u$ be the entropy solution of the initial-boundary value problem (4.1) and $u_{\Delta t}$ the numerical approximation given by (4.2) where we take $\lambda$ constant. Then we have the following convergence rate estimate:

$$
\left\|u(\cdot, T)-u_{\Delta t}(\cdot, T)\right\|_{\mathrm{L}^{1}\left(\mathbb{R}^{-}\right)} \leq C \sqrt{\Delta x}
$$

for some constant $C$ independent of $\Delta x$.

Proof. The numerical solution $u_{\Delta t}$ is Lipschitz continuous in time and TVD, and therefore satisfies

$$
\begin{aligned}
\nu_{0}\left(u_{\Delta t}, \varepsilon_{0}\right), \nu_{T}\left(u_{\Delta t}, \varepsilon_{0}\right) & \leq C \operatorname{TV}\left(u_{0}\right)\left(\varepsilon_{0}+\Delta t\right) \\
\text { and } \quad \mu\left(u_{\Delta t}(\cdot, 0), \varepsilon\right), \mu\left(u_{\Delta t}(\cdot, T), \varepsilon\right) & \leq \operatorname{TV}\left(u_{0}\right) \varepsilon
\end{aligned}
$$

Thus, taking into consideration Lemmas 4.2 and 4.3, we have

$$
\begin{aligned}
& \left\|u(\cdot, T)-u_{\Delta t}(\cdot, T)\right\|_{\mathrm{L}^{1}\left(\mathbb{R}^{-}\right)} \\
& +\int_{0}^{T} \int_{\mathbb{R}^{-}} \int_{0}^{T}\left(q\left(u(0-, t), u_{\Delta t}(y, s)\right)+q\left(u_{\Delta t}(0-, t), u(y, s)\right)\right) \varphi(0, t, y, s) \mathrm{d} t \mathrm{~d} y \mathrm{~d} s \\
& \quad \leq\left\|u_{0}-u_{\Delta t}(\cdot, 0)\right\|_{\mathrm{L}^{1}\left(\mathbb{R}^{-}\right)}+C\left(\Delta x+\Delta t+\varepsilon+\varepsilon_{0}+\frac{\Delta x}{\varepsilon}+\frac{\Delta x}{\varepsilon_{0}}+\frac{\Delta t}{\varepsilon_{0}}\right) .
\end{aligned}
$$

Because of our choice of discretizing the initial datum as $u_{j}^{0}=\frac{1}{\Delta x} \int_{\mathcal{C}_{j}} u_{0}(x) \mathrm{d} x$ we have $\left\|u_{\Delta t}(\cdot, 0)-u_{0}\right\|_{\mathrm{L}^{1}\left(\mathbb{R}^{-}\right)} \leq C \operatorname{TV}\left(u_{0}\right) \Delta x$. Now, in order to get a convergence rate, 
we take $\lambda=\frac{\Delta t}{\Delta x}$ constant and minimize the right-hand side of the above estimate for $\varepsilon$ and $\varepsilon_{0}$. This yields $\varepsilon=\varepsilon_{0}=\sqrt{\Delta x}$ and hence

$$
\begin{array}{r}
\left\|u(\cdot, T)-u_{\Delta t}(\cdot, T)\right\|_{\mathrm{L}^{1}\left(\mathbb{R}^{-}\right)} \\
+\int_{0}^{T} \int_{\mathbb{R}^{-}} \int_{0}^{T}\left(q\left(u(0-, t), u_{\Delta t}(y, s)\right)+q\left(u_{\Delta t}(0-, t), u(y, s)\right)\right) \varphi(0, t, y, s) \mathrm{d} t \mathrm{~d} y \mathrm{~d} s \\
\leq C \sqrt{\Delta x} .
\end{array}
$$

Using the monotonicity of $g$ we find

$$
q(u, v)=|g(u)-g(v)| \geq 0
$$

and thus the integral term in (4.7) is nonnegative which concludes the proof.

4.2. Convergence rate estimates on $\mathbb{R}^{+}$. As a second step we now consider the initial-boundary value problem

$$
\begin{aligned}
u_{t}+f(u)_{x}=0, & (x, t) \in \mathbb{R}^{+} \times(0, T), \\
u(x, 0)=u_{0}(x), & x \in \mathbb{R}^{+}, \\
u(0, t)=f^{-1}(g(u(0-, t)), & t \in(0, T)
\end{aligned}
$$

and the numerical scheme

$$
\begin{gathered}
u_{j}^{n+1}=u_{j}^{n}-\lambda\left(f\left(u_{j}^{n}\right)-f\left(u_{j-1}^{n}\right)\right), \quad j \geq 1, n \geq 0 \\
u_{j}^{0}=\frac{1}{\Delta x} \int_{\mathcal{C}_{j}} u_{0}(x) \mathrm{d} x, \quad j \geq 0, \\
u_{0}^{n}=f^{-1}\left(g\left(u_{-1}^{n}\right)\right), \quad n \geq 1
\end{gathered}
$$

where the boundary data is given in terms of $u(0,-, t)$ and $u_{-1}^{n}$ respectively and those are known from the previous section. Note that again we have a discrete entropy inequality of the form

$$
D_{+}^{t} \eta_{j}^{n}+D_{-} q_{j}^{n} \leq 0, \quad n \geq 1, j \geq 1 .
$$

Definition 4.5 (Entropy solution on $\mathbb{R}^{+}$). We say $u \in \mathcal{C}\left([0, T] ; \mathrm{L}^{1}\left(\mathbb{R}^{+}\right)\right) \cap$ $\mathrm{L}^{\infty}\left(\mathbb{R}^{+} \times(0, T)\right)$ is an entropy solution of Equation (4.8) if for all $c \in \mathbb{R}$,

$$
\begin{aligned}
\int_{0}^{T} \int_{\mathbb{R}^{+}}\left(|u-c| \varphi_{t}+|f(u)-f(c)| \varphi_{x}\right) \mathrm{d} x \mathrm{~d} t-\int_{\mathbb{R}^{+}}|u(x, T)-c| \varphi(x, T) \mathrm{d} x \\
\left.\quad+\int_{\mathbb{R}^{+}}\left|u_{0}(x)-c\right| \varphi(x, 0)\right) \mathrm{d} x+\int_{0}^{T}|f(u(0+, t))-f(c)| \varphi(0, t) \mathrm{d} t \geq 0
\end{aligned}
$$

for all nonnegative $\varphi \in \mathcal{C}^{\infty}([0, \infty) \times[0, T])$ and

$$
f(u(0+, t))=g(u(0-, t))
$$

holds for almost every $t \in(0, T)$.

Before we calculate convergence rates on $\mathbb{R}^{+}$we need two auxiliary lemmas that are consequences of the monotonicity of the flux. 
Lemma 4.6 (Bound on the temporal total variation). If the numerical scheme (4.9) satisfies the CFL condition (3.2) the temporal variation of the numerical solution is bounded, specifically, for every $j \in \mathbb{Z}$ we have

$$
\sum_{n=0}^{M}\left|u_{j}^{n+1}-u_{j}^{n}\right| \leq C \operatorname{TV}\left(u_{0}\right)
$$

where $\operatorname{TV}\left(u_{0}\right)$ refers to the total variation of $u_{0}$ on the whole real line.

Proof. Let first $j \geq 1$. Using the CFL condition (3.2) and the monotonicity of the flux, i.e., $f^{\prime}>0$, we find that

$$
\begin{aligned}
\left|u_{j}^{n}-u_{j-1}^{n}-\lambda\left(f\left(u_{j}^{n}\right)-f\left(u_{j-1}^{n}\right)\right)\right| & =\left|u_{j}^{n}-u_{j-1}^{n}-\lambda f^{\prime}\left(u^{*}\right)\left(u_{j}^{n}-u_{j-1}^{n}\right)\right| \\
& =\left(1-\lambda f^{\prime}\left(u^{*}\right)\right)\left|u_{j}^{n}-u_{j-1}^{n}\right| \\
& =\left|u_{j}^{n}-u_{j-1}^{n}\right|-\lambda f^{\prime}\left(u^{*}\right)\left|u_{j}^{n}-u_{j-1}^{n}\right| \\
& =\left|u_{j}^{n}-u_{j-1}^{n}\right|-\lambda\left|f\left(u_{j}^{n}\right)-f\left(u_{j-1}^{n}\right)\right|
\end{aligned}
$$

and hence

$$
\begin{aligned}
\left|u_{j}^{n+1}-u_{j-1}^{n+1}\right| & =\left|u_{j}^{n}-u_{j-1}^{n}-\lambda\left(f\left(u_{j}^{n}\right)-f\left(u_{j-1}^{n}\right)\right)+\lambda\left(f\left(u_{j-1}^{n}\right)-f\left(u_{j-2}^{n}\right)\right)\right| \\
& \leq\left|u_{j}^{n}-u_{j-1}^{n}-\lambda\left(f\left(u_{j}^{n}\right)-f\left(u_{j-1}^{n}\right)\right)\right|+\lambda\left|f\left(u_{j-1}^{n}\right)-f\left(u_{j-2}^{n}\right)\right| \\
& =\left|u_{j}^{n}-u_{j-1}^{n}\right|-\lambda\left|f\left(u_{j}^{n}\right)-f\left(u_{j-1}^{n}\right)\right|+\lambda\left|f\left(u_{j-1}^{n}\right)-f\left(u_{j-2}^{n}\right)\right| \\
& =\left|u_{j}^{n}-u_{j-1}^{n}\right|-\left|u_{j}^{n+1}-u_{j}^{n}\right|+\left|u_{j-1}^{n+1}-u_{j-1}^{n}\right|
\end{aligned}
$$

where we have used the definition of the numerical scheme (4.9) in the last step. Taking the sum over $n=0, \ldots, M-1$ yields

$$
\sum_{n=0}^{M-1}\left|u_{j}^{n+1}-u_{j-1}^{n+1}\right| \leq \sum_{n=0}^{M-1}\left|u_{j}^{n}-u_{j-1}^{n}\right|-\sum_{n=0}^{M-1}\left|u_{j}^{n+1}-u_{j}^{n}\right|+\sum_{n=0}^{M-1}\left|u_{j-1}^{n+1}-u_{j-1}^{n}\right|
$$

where we can cancel equal terms to get

$$
\left|u_{j}^{M}-u_{j-1}^{M}\right| \leq\left|u_{j}^{0}-u_{j-1}^{0}\right|-\sum_{n=0}^{M-1}\left|u_{j}^{n+1}-u_{j}^{n}\right|+\sum_{n=0}^{M-1}\left|u_{j-1}^{n+1}-u_{j-1}^{n}\right| .
$$

Because of the CFL condition (3.2) we have

$$
\left|u_{j}^{M+1}-u_{j}^{M}\right|=\lambda\left|f\left(u_{j}^{M}\right)-f\left(u_{j-1}^{M}\right)\right|=\lambda f^{\prime}\left(u^{*}\right)\left|u_{j}^{M}-u_{j-1}^{M}\right| \leq\left|u_{j}^{M}-u_{j-1}^{M}\right|
$$

which together with (4.11) yields

$$
\left|u_{j}^{M+1}-u_{j}^{M}\right| \leq\left|u_{j}^{0}-u_{j-1}^{0}\right|-\sum_{n=0}^{M-1}\left|u_{j}^{n+1}-u_{j}^{n}\right|+\sum_{n=0}^{M-1}\left|u_{j-1}^{n+1}-u_{j-1}^{n}\right|
$$

and thus

$$
\sum_{n=0}^{M}\left|u_{j}^{n+1}-u_{j}^{n}\right| \leq\left|u_{j}^{0}-u_{j-1}^{0}\right|+\sum_{n=0}^{M-1}\left|u_{j-1}^{n+1}-u_{j-1}^{n}\right|
$$


By substituting $f$ with $g$ in the above calculations, the estimate (4.12) also holds for $j<0$. The estimate (4.12) now allows us to bound the temporal variation of the numerical scheme by the total variation of the initial datum in the following way. If $j>M$ or $j<0$ we can use the estimate (4.12) iteratively to get

$$
\sum_{n=0}^{M}\left|u_{j}^{n+1}-u_{j}^{n}\right| \leq \sum_{i=j-M+1}^{j}\left|u_{i}^{0}-u_{i-1}^{0}\right|+\left|u_{j-M}^{1}-u_{j-M}^{0}\right| .
$$

Using the definition of the scheme (4.9), we get

$$
\left|u_{j-M}^{1}-u_{j-M}^{0}\right|=\lambda\left|f\left(u_{j-M}^{0}\right)-f\left(u_{j-M-1}^{0}\right)\right| \leq C \lambda\left|u_{j-M}^{0}-u_{j-M-1}^{0}\right|
$$

such that we have

$$
\sum_{n=0}^{M}\left|u_{j}^{n+1}-u_{j}^{n}\right| \leq C \sum_{i=j-M}^{j}\left|u_{i}^{0}-u_{i-1}^{0}\right| \leq C \operatorname{TV}\left(u_{0}\right) .
$$

If on the other hand $0 \leq j \leq M$ we get

$$
\sum_{n=0}^{M}\left|u_{j}^{n+1}-u_{j}^{n}\right| \leq \sum_{i=1}^{j}\left|u_{i}^{0}-u_{i-1}^{0}\right|+\sum_{n=0}^{M-j}\left|u_{0}^{n+1}-u_{0}^{n}\right| .
$$

Using the definition of $u_{0}^{n}$ in (4.9) and applying (4.12) iteratively again, we get

$$
\begin{aligned}
\sum_{n=0}^{M-j}\left|u_{0}^{n+1}-u_{0}^{n}\right| & =\sum_{n=0}^{M-j}\left|f^{-1}\left(g\left(u_{-1}^{n+1}\right)\right)-f^{-1}\left(g\left(u_{-1}^{n}\right)\right)\right| \\
& \leq \frac{C}{\alpha} \sum_{n=0}^{M-j}\left|u_{-1}^{n+1}-u_{-1}^{n}\right| \\
& \leq C \sum_{i=-1-(M-j)}^{-1}\left|u_{i}^{0}-u_{i-1}^{0}\right|
\end{aligned}
$$

such that we have

$$
\sum_{n=0}^{M}\left|u_{j}^{n+1}-u_{j}^{n}\right| \leq C \sum_{i=-1-(M-j)}^{j}\left|u_{i}^{0}-u_{i-1}^{0}\right| \leq C \operatorname{TV}\left(u_{0}\right)
$$

which concludes the proof.

Lemma 4.7. Let $u$ be the entropy solution of (4.8) and assume $f^{\prime}>0$. Then $f(u)$ is Lipschitz continuous in space, in the sense that

$$
\int_{0}^{T}|f(u(x, t))-f(u(y, t))| \mathrm{d} t \leq C|x-y| \quad \text { for all } x, y \in \mathbb{R}^{+} .
$$

Proof. Since $u$ is bounded, we can assume that $f^{\prime} \geq \alpha>0$. Thus the flux is invertible with Lipschitz continuous inverse. By setting $w=f(u)$ and $h=f^{-1}$ we find that $w$ satisfies

$$
w_{x}+h(w)_{t}=0, \quad(t, x) \in(0, T) \times \mathbb{R}^{+} .
$$


By the standard theory for conservation laws (with the roles of $x$ and $t$ reversed) adapted to the bounded domain $[0, T]$ we see that $w$ is Lipschitz continuous in $x$ with values in $\mathrm{L}^{1}(0, \mathrm{~T})$, i.e.,

$$
\int_{0}^{T}|f(u(x, t))-f(u(y, t))| \mathrm{d} t=\int_{0}^{T}|w(x, t)-w(y, t)| \mathrm{d} t \leq C|x-y|,
$$

cf. [15, Thm. 2.15] or [31, Lem. 4]. Note that an application of [31, Lem. 4] requires, in particular, a temporal total variation bound of $u(0+, t)$ which follows from Lemma 4.6 on a discrete level and caries over in the limit.

We will now describe how to modify the steps in Section 4.1 in order to get a convergence rate on $\mathbb{R}^{+}$. We start by defining

$$
\begin{aligned}
L(u, c, \varphi)= & \int_{0}^{T} \int_{\mathbb{R}^{+}}\left(|u-c| \varphi_{t}+q(u, c) \varphi_{x}\right) \mathrm{d} x \mathrm{~d} t-\int_{\mathbb{R}^{+}}|u(x, T)-c| \varphi(x, T) \mathrm{d} x \\
& +\int_{\mathbb{R}^{+}}\left|u_{0}(x)-c\right| \varphi(x, 0) \mathrm{d} x+\int_{0}^{T} q(u(0+, t), c) \varphi(0, t) \mathrm{d} t
\end{aligned}
$$

and

$$
\Lambda_{\varepsilon, \varepsilon_{0}}(u, v)=\int_{0}^{T} \int_{\mathbb{R}^{+}} L(u, v(y, s), \varphi(\cdot, \cdot, y, s)) \mathrm{d} y \mathrm{~d} s
$$

where again $\varphi=\omega_{\varepsilon}(x-y) \omega_{\varepsilon_{0}}(t-s)$.

Lemma 4.8 (Kuznetsov-type lemma). Let $u$ be the entropy solution of (4.8). Then, for any function $v:[0, T] \rightarrow\left(\mathrm{L}^{1} \cap \mathrm{BV}\right)\left(\mathbb{R}^{+}\right)$such that the one-sided limits $v(t \pm)$ exist in $\mathrm{L}^{1}$, we have

$$
\begin{aligned}
& \| u(\cdot, T)-v(\cdot, T)\left\|_{\mathrm{L}^{1}\left(\mathbb{R}^{+}\right)} \leq\right\| u_{0}-v(\cdot, 0) \|_{\mathrm{L}^{1}\left(\mathbb{R}^{+}\right)}-\Lambda_{\varepsilon, \varepsilon_{0}}(v, u) \\
&\left.\left.+C\left(\varepsilon+\varepsilon_{0}+\nu_{T}\left(v, \varepsilon_{0}\right)+\nu_{0}\left(v, \varepsilon_{0}\right)+\mu(v(\cdot, T), \varepsilon)\right)+\mu(v(\cdot, 0), \varepsilon)\right)\right) \\
& \quad+\int_{0}^{T} \int_{\mathbb{R}^{+}} \int_{0}^{T}(q(u(0+, t), v(y, s))+q(v(0+, t), u(y, s))) \varphi(0, t, y, s) \mathrm{d} t \mathrm{~d} y \mathrm{~d} s
\end{aligned}
$$

for some constant $C$ independent of $\varepsilon$ and $\varepsilon_{0}$.

Note that this time the term involving $q$ is on the right hand side of the inequality.

Proof. The proof follows the same steps, mutatis mutandis, as the proof of the Kuznetsov-type lemma 4.2 on $\mathbb{R}^{-}$.

Lemma 4.9. The estimate

$$
-\Lambda_{\varepsilon, \varepsilon_{0}}\left(u_{\Delta t}, u\right) \leq C\left(\Delta x+\frac{\Delta x}{\varepsilon}+\frac{\Delta x}{\varepsilon_{0}}+\frac{\Delta t}{\varepsilon_{0}}\right)
$$

holds for some constant $C$ independent of $\Delta x, \Delta t, \varepsilon$, and $\varepsilon_{0}$.

Note that the right-hand side of the inequality contains the term $\frac{\Delta x}{\varepsilon_{0}}$ which was not present in Lemma 4.3, but will not change the overall convergence rate. 
Proof. Using summation by parts and the discrete entropy inequality (4.10), $D_{+}^{t} \eta_{j}^{n}+D_{-} q_{j}^{n} \leq 0$ for $j \geq 1$, we find

$$
\begin{aligned}
- & L\left(u_{\Delta t}, u, \varphi\right) \\
= & -\sum_{n=0}^{M} \sum_{j=0}^{\infty}\left(\eta_{j}^{n} \iint_{\mathcal{C}_{j}^{n}} \varphi_{t} \mathrm{~d} x \mathrm{~d} t+q_{j}^{n} \iint_{\mathcal{C}_{j}^{n}} \varphi_{x} \mathrm{~d} x \mathrm{~d} t\right) \\
& -\sum_{j=0}^{\infty} \eta_{j}^{0} \int_{\mathcal{C}_{j}} \varphi^{0} \mathrm{~d} x+\sum_{j=0}^{\infty} \eta_{j}^{M+1} \int_{\mathcal{C}_{j}} \varphi^{M+1} \mathrm{~d} x-\sum_{n=0}^{M} q_{0}^{n} \int_{\mathcal{C}^{n}} \varphi_{-1 / 2} \mathrm{~d} t \\
= & \sum_{n=0}^{M} \sum_{j=0}^{\infty}\left(D_{+}^{t} \eta_{j}^{n} \iint_{\mathcal{C}_{j}^{n}} \varphi^{n+1} \mathrm{~d} x \mathrm{~d} t+D_{-} q_{j+1}^{n} \iint_{\mathcal{C}_{j}^{n}} \varphi_{j+\frac{1}{2}} \mathrm{~d} x \mathrm{~d} t\right) \\
\leq & \sum_{n=0}^{M}\left(D_{+}^{t} \eta_{0}^{n} \iint_{\mathcal{C}_{0}^{n}} \varphi^{n+1} \mathrm{~d} x \mathrm{~d} t+\sum_{j=0}^{\infty} D_{-} q_{j+1}^{n} \iint_{\mathcal{C}_{j}^{n}} \varphi_{j+1 / 2} \mathrm{~d} x \mathrm{~d} t\right. \\
& \left.\quad-\sum_{j=1}^{\infty} D_{-} q_{j}^{n} \iint_{\mathcal{C}_{j}^{n}} \varphi^{n+1} \mathrm{~d} x \mathrm{~d} t\right) \\
= & \sum_{n=0}^{M} \underbrace{D_{+}^{t} \eta_{0}^{n} \iint_{\mathcal{C}_{0}^{n}} \varphi^{n+1} \mathrm{~d} x \mathrm{~d} t}_{=: \mathbb{D}^{\mathbf{n}}}+\sum_{n=0}^{M} \sum_{j=1}^{\infty} \underbrace{D_{-} q_{j}^{n} \iint_{\mathcal{C}_{j}^{n}}\left(\varphi_{j-1 / 2}-\varphi^{n+1}\right) \mathrm{d} x \mathrm{~d} t}_{=: \mathbb{E}_{\mathbf{j}}^{n}}
\end{aligned}
$$

where we have used the notation $\varphi^{n}=\varphi\left(x, t^{n}, y, s\right)$ and $\varphi_{j+1 / 2}=\varphi\left(x_{j+1 / 2}, t, y, s\right)$.

Concerning the term $\mathbf{D}^{\mathbf{n}}$, using summation by parts again, we find

$$
\begin{aligned}
& \sum_{n=0}^{M} \int_{0}^{T} \int_{\mathbb{R}^{+}} \mathbf{D}^{\mathbf{n}} \mathrm{d} y \mathrm{~d} s \\
& =\int_{0}^{T} \int_{\mathbb{R}^{+}}\left(\eta_{0}^{M+1} \int_{\mathcal{C}_{0}} \varphi^{M+1} \mathrm{~d} x-\eta_{0}^{0} \int_{\mathcal{C}_{0}} \varphi^{0} \mathrm{~d} x-\sum_{n=0}^{M} \eta_{0}^{n} \iint_{\mathcal{C}_{0}^{n}} D_{+}^{t} \varphi^{n} \mathrm{~d} x \mathrm{~d} t\right) \mathrm{d} y \mathrm{~d} s .
\end{aligned}
$$

Here, using the boundedness of $\eta^{\prime}$ and the properties of the mollifiers (4.5), the boundary terms can be estimated as follows:

$$
\int_{0}^{T} \int_{\mathbb{R}^{+}} \underbrace{\eta_{0}^{M+1}}_{\leq C\left\|u_{0}\right\|_{\infty}} \int_{\mathcal{C}_{0}} \varphi^{M+1} \mathrm{~d} x \mathrm{~d} y \mathrm{~d} s \leq C \Delta x
$$

and similarly

$$
\int_{0}^{T} \int_{\mathbb{R}^{+}} \eta_{0}^{0} \int_{\mathcal{C}_{0}} \varphi^{0} \mathrm{~d} x \mathrm{~d} y \mathrm{~d} s \leq C \Delta x .
$$


For the remaining term, we can proceed in the following way

$$
\begin{aligned}
& \sum_{n=0}^{M} \int_{0}^{T} \int_{\mathbb{R}^{+}} \underbrace{\eta_{0}^{n}}_{\leq C\left\|u_{0}\right\|_{\infty}} \iint_{\mathcal{C}_{0}^{n}} D_{+}^{t} \varphi^{n} \mathrm{~d} x \mathrm{~d} t \mathrm{~d} y \mathrm{~d} s \\
& \quad \leq C \sum_{n=0}^{M} \int_{0}^{T} \int_{\mathbb{R}^{+}} \frac{1}{\Delta t} \iint_{\mathcal{C}_{0}^{n}} \int_{\mathcal{C}^{n}}\left|\omega_{\varepsilon_{0}}^{\prime}(\tau-s)\right| \mathrm{d} \tau \omega_{\varepsilon}(x-y) \mathrm{d} x \mathrm{~d} t \mathrm{~d} y \mathrm{~d} s \\
& \quad \leq C \sum_{n=0}^{M} \frac{\Delta x \Delta t^{2}}{\Delta t \varepsilon_{0}} \\
& \quad \leq C \frac{\Delta x}{\varepsilon_{0}} .
\end{aligned}
$$

We split the term involving $\mathbf{E}_{\mathbf{j}}^{\mathbf{n}}$ as follows:

$$
\begin{aligned}
\sum_{n=0}^{M} \sum_{j=1}^{\infty} \mathbf{E}_{\mathbf{j}}^{\mathbf{n}} \leq & \sum_{n=0}^{M} \sum_{j=1}^{\infty} \underbrace{\left|D_{-} q_{j}^{n}\right| \iint_{\mathcal{C}_{j}^{n}} \int_{x_{j+1} / 2}^{x}\left|\varphi_{x}(z, t)\right| \mathrm{d} z \mathrm{~d} x \mathrm{~d} t}_{=: \mathbf{F}_{\mathbf{j}}^{\mathbf{n}}} \\
& +\sum_{n=0}^{M} \sum_{j=1}^{\infty} \underbrace{\left|D_{-} q_{j}^{n}\right| \iint_{\mathcal{C}_{j}^{n}} \int_{t}^{t^{n+1}}\left|\varphi_{t}(x, \tau)\right| \mathrm{d} \tau \mathrm{d} x \mathrm{~d} t}_{=: \mathbf{G}_{\mathbf{j}}^{\mathbf{n}}}
\end{aligned}
$$

For the first term, using the properties of the mollifiers (4.5) and the Lipschitz continuity of $f$, we find

$$
\begin{aligned}
\int_{0}^{T} \int_{\mathbb{R}^{+}} & \sum_{n=0}^{M} \sum_{j=1}^{\infty} \mathbf{F}_{\mathbf{j}}^{\mathbf{n}} \mathrm{d} y \mathrm{~d} s \\
& =\sum_{n=0}^{M} \sum_{j=1}^{\infty}\left|D_{-} q_{j}^{n}\right| \int_{0}^{T} \int_{\mathbb{R}^{+}} \iint_{\mathcal{C}_{j}^{n}} \int_{x_{j+1 / 2}}^{x}\left|\omega_{\varepsilon}^{\prime}(z-y)\right| \mathrm{d} z \omega_{\varepsilon_{0}}(t-s) \mathrm{d} x \mathrm{~d} t \mathrm{~d} y \mathrm{~d} s \\
& \leq \sum_{n=0}^{M} \sum_{j=1}^{\infty} \frac{C}{\Delta x}\left|u_{j}^{n}-u_{j-1}^{n}\right| \frac{\Delta x^{2} \Delta t}{\varepsilon} \\
& \leq C \operatorname{TV}\left(u_{0}\right) \frac{\Delta x}{\varepsilon}
\end{aligned}
$$

and similarly

$$
\int_{0}^{T} \int_{\mathbb{R}^{+}} \sum_{n=0}^{M} \sum_{j=1}^{\infty} \mathbf{G}_{\mathbf{j}}^{\mathbf{n}} \mathrm{d} y \mathrm{~d} s \leq C \operatorname{TV}\left(u_{0}\right) \frac{\Delta t}{\varepsilon_{0}} .
$$

Thus, we have

$$
-\Lambda_{\varepsilon, \varepsilon_{0}}\left(u_{\Delta t}, u\right) \leq C\left(\Delta x+\frac{\Delta x}{\varepsilon}+\frac{\Delta x}{\varepsilon_{0}}+\frac{\Delta t}{\varepsilon_{0}}\right)
$$

which concludes the proof. 
THEOREM 4.10 (Convergence rate on $\mathbb{R}^{+}$). Let $u$ be the entropy solution of the initial-boundary value problem (4.8) and $u_{\Delta t}$ the numerical approximation given by (4.9). Then we have the following convergence rate estimate:

$$
\left\|u(\cdot, T)-u_{\Delta t}(\cdot, T)\right\|_{\mathrm{L}^{1}\left(\mathbb{R}^{+}\right)} \leq C \sqrt{\Delta x}
$$

for some constant $C$ independent of $\Delta x$.

Proof. The numerical solution $u_{\Delta t}$ is Lipschitz continuous in time and TVD (for the TVD property of conservation laws on bounded domains see [31, Lem. 2]), and therefore satisfies

$$
\begin{aligned}
\nu_{0}\left(u_{\Delta t}, \varepsilon_{0}\right), \nu_{T}\left(u_{\Delta t}, \varepsilon_{0}\right) & \leq C \operatorname{TV}\left(u_{0}\right)\left(\varepsilon_{0}+\Delta t\right) \\
\text { and } \quad \mu\left(u_{\Delta t}(\cdot, 0), \varepsilon\right), \mu\left(u_{\Delta t}(\cdot, T), \varepsilon\right) & \leq \operatorname{TV}\left(u_{0}\right) \varepsilon
\end{aligned}
$$

Thus, taking into consideration Lemmas 4.2 and 4.3, we have

$$
\begin{aligned}
\left\|u(\cdot, T)-u_{\Delta t}(\cdot, T)\right\|_{\mathrm{L}^{1}\left(\mathbb{R}^{+}\right) \leq} & \left\|u_{0}-u_{\Delta t}(\cdot, 0)\right\|_{\mathrm{L}^{1}\left(\mathbb{R}^{+}\right)} \\
& +C\left(\Delta x+\Delta t+\varepsilon+\varepsilon_{0}+\frac{\Delta x}{\varepsilon}+\frac{\Delta x}{\varepsilon_{0}}+\frac{\Delta t}{\varepsilon_{0}}\right)+\mathbf{C}
\end{aligned}
$$

where

$$
\mathbf{C}=\int_{0}^{T} \int_{\mathbb{R}^{+}} \int_{0}^{T}\left(q\left(u(0+, t), u_{\Delta t}(y, s)\right)+q\left(u_{\Delta t}(0+, t), u(y, s)\right)\right) \varphi(0, t, y, s) \mathrm{d} t \mathrm{~d} y \mathrm{~d} s
$$

Because of our choice of discretizing the initial datum as $u_{j}^{0}=\frac{1}{\Delta x} \int_{\mathcal{C}_{j}} u_{0}(x) \mathrm{d} x$ we have $\left\|u_{\Delta t}(\cdot, 0)-u_{0}\right\|_{\mathrm{L}^{1}\left(\mathbb{R}^{+}\right)} \leq C \operatorname{TV}\left(u_{0}\right) \Delta x$ and thus it remains to estimate the term

$$
\begin{aligned}
\mathbf{C}=\int_{0}^{T} \int_{\mathbb{R}^{+}} \int_{0}^{T}(\underbrace{\left|f(u(0+, t))-f\left(u_{\Delta t}(y, s)\right)\right|}_{=: \mathbf{H}} \\
+\underbrace{\left|f\left(u_{\Delta t}(0+, t)\right)-f(u(y, s))\right|}_{=: \mathbf{J}}) \varphi(0, t, y, s) \mathrm{d} t \mathrm{~d} y \mathrm{~d} s .
\end{aligned}
$$

Here, we split

$$
\mathbf{H} \leq \underbrace{\left|f(u(0+, t))-f\left(u_{\Delta t}(0+, s)\right)\right|}_{\mathbf{H}_{\mathbf{1}}}+\underbrace{\left|f\left(u_{\Delta t}(0+, s)\right)-f\left(u_{\Delta t}(y, s)\right)\right|}_{\mathbf{H}_{\mathbf{2}}} .
$$

Using the Rankine-Hugoniot condition, the term $\mathbf{H}_{\mathbf{1}}$ can be estimated as follows:

$$
\begin{aligned}
\mathbf{H}_{\mathbf{1}} & =\left|f(u(0+, t))-f\left(u_{\Delta t}(0+, s)\right)\right| \\
& =\left|g(u(0-, t))-g\left(u_{\Delta t}(0-, s)\right)\right| \\
& \leq\left|g(u(0-, t))-g\left(u_{\Delta t}(y, s)\right)\right|+\left|g\left(u_{\Delta t}(y, s)\right)-g\left(u_{\Delta t}(0-, s)\right)\right| .
\end{aligned}
$$

Because $\mathbf{H}_{\mathbf{1}}$ does not depend on $y$ we can use the symmetry of $\varphi$ with respect to $y$ 
and the estimate (4.7) to get

$$
\begin{aligned}
& \int_{0}^{T} \int_{\mathbb{R}^{+}} \int_{0}^{T} \mathbf{H}_{\mathbf{1}} \varphi(0, t, y, s) \mathrm{d} t \mathrm{~d} y \mathrm{~d} s \\
& =\int_{0}^{T} \int_{\mathbb{R}^{-}} \int_{0}^{T} \mathbf{H}_{\mathbf{1}} \varphi(0, t, y, s) \mathrm{d} t \mathrm{~d} y \mathrm{~d} s \\
& \leq \int_{0}^{T} \int_{\mathbb{R}^{-}} \int_{0}^{T}\left|g(u(0-, t))-g\left(u_{\Delta t}(y, s)\right)\right| \varphi(0, t, y, s) \mathrm{d} t \mathrm{~d} y \mathrm{~d} s \\
& \quad+\int_{0}^{T} \int_{\mathbb{R}^{-}} \int_{0}^{T}\left|g\left(u_{\Delta t}(y, s)\right)-g\left(u_{\Delta t}(0-, s)\right)\right| \varphi(0, t, y, s) \mathrm{d} t \mathrm{~d} y \mathrm{~d} s \\
& \leq C \sqrt{\Delta x}+\int_{0}^{T} \int_{\mathbb{R}^{-}} \int_{0}^{T}\left|g\left(u_{\Delta t}(y, s)\right)-g\left(u_{\Delta t}(0-, s)\right)\right| \varphi(0, t, y, s) \mathrm{d} t \mathrm{~d} y \mathrm{~d} s .
\end{aligned}
$$

Using the identity

$$
\left|g\left(u_{i+1}^{n}\right)-g\left(u_{i}^{n}\right)\right|=\frac{1}{\lambda}\left|u_{i+1}^{n+1}-u_{i+1}^{n}\right|
$$

and setting $N=\left\lceil\frac{\varepsilon}{\Delta x}\right\rceil$, we can employ Lemma 4.6 to estimate the integral term in the foregoing estimate as follows:

$$
\begin{aligned}
& \int_{0}^{T} \int_{\mathbb{R}^{-}} \int_{0}^{T}\left|g\left(u_{\Delta t}(y, s)\right)-g\left(u_{\Delta t}(0-, s)\right)\right| \varphi(0, t, y, s) \mathrm{d} t \mathrm{~d} y \mathrm{~d} s \\
& =\sum_{n=0}^{M} \sum_{j=-N}^{-1}\left|g\left(u_{j}^{n}\right)-g\left(u_{-1}^{n}\right)\right| \int_{0}^{T} \iint_{\mathcal{C}_{j}^{n}} \omega_{\varepsilon_{0}}(t-s) \omega_{\varepsilon}(y) \mathrm{d} t \mathrm{~d} y \mathrm{~d} s \\
& \leq C \frac{\Delta t \Delta x}{\varepsilon} \sum_{n=0}^{M} \sum_{j=-N}^{-1} \sum_{i=j}^{-2} \underbrace{\left|g\left(u_{i+1}^{n}\right)-g\left(u_{i}^{n}\right)\right|}_{=\frac{1}{\lambda\left|u_{i+1}^{n+1}-u_{i+1}^{n}\right|}} \\
& \leq C \frac{\Delta t \Delta x}{\varepsilon} \sum_{j=-N}^{-1} \sum_{i=j}^{-2} \sum_{n=0}^{M}\left|u_{i+1}^{n+1}-u_{i+1}^{n}\right| \\
& \leq C \frac{\Delta t \Delta x}{\varepsilon} \sum_{j=-N}^{-1}(-j) \\
& \leq C \frac{\Delta t \Delta x}{\varepsilon} \frac{N(N+1)}{2} \\
& \leq C \Delta t\left(\frac{\varepsilon}{\Delta x}+1\right) \\
& \leq C(\varepsilon+\Delta t) .
\end{aligned}
$$

The term involving $\mathbf{H}_{\mathbf{2}}$ can be estimated analogously. Then it remains to treat the integral involving $\mathbf{J}$. We split $\mathbf{J}$ as follows

$$
\mathbf{J} \leq \underbrace{\left|f\left(u_{\Delta t}(0+, t)\right)-f(u(0+, s))\right|}_{\mathbf{J}_{\mathbf{1}}}+\underbrace{|f(u(0+, s))-f(u(y, s))|}_{\mathbf{J}_{\mathbf{2}}}
$$


and note that the $\mathbf{J}_{\mathbf{1}}$ is the same as $\mathbf{H}_{\mathbf{1}}$. Lastly, with the help of Lemma 4.7 we find

$$
\begin{aligned}
\int_{0}^{T} \int_{\mathbb{R}^{+}} \int_{0}^{T} & \mathbf{J}_{\mathbf{2}} \varphi(0, t, y, s) \mathrm{d} t \mathrm{~d} y \mathrm{~d} s \\
& =\int_{0}^{T} \int_{\mathbb{R}^{+}} \int_{0}^{T}|f(u(0+, s))-f(u(y, s))| \varphi(0, t, y, s) \mathrm{d} t \mathrm{~d} y \mathrm{~d} s \\
& \leq \frac{1}{\varepsilon} \int_{0}^{\varepsilon} \underbrace{\int_{0}^{T}|f(u(0+, s))-f(u(y, s))| \mathrm{d} s}_{\leq C|y|} \mathrm{d} y \\
& \leq \frac{C}{\varepsilon} \int_{0}^{\varepsilon}|y| \mathrm{d} y \\
& \leq C \varepsilon .
\end{aligned}
$$

Finally, we have

$$
\left\|u(\cdot, T)-u_{\Delta t}(\cdot, T)\right\|_{\mathrm{L}^{1}\left(\mathbb{R}^{+}\right)} \leq C\left(\Delta x+\Delta t+\varepsilon+\varepsilon_{0}+\frac{\Delta x}{\varepsilon}+\frac{\Delta x}{\varepsilon_{0}}+\frac{\Delta t}{\varepsilon_{0}}\right) .
$$

In order to get a convergence rate, again we take $\lambda=\frac{\Delta t}{\Delta x}$ constant and minimize the right-hand side of the above estimate for $\varepsilon$ and $\varepsilon_{0}$. This yields $\varepsilon=\varepsilon_{0}=\sqrt{\Delta x}$ which concludes the proof.

4.3. Convergence rate estimates on $(0, L)$. By restricting the solution $u$ and the numerical approximation $u_{\Delta t}$ to a bounded interval $(0, L)$ Theorem 4.10 and the estimate (4.7) yield a convergence rate on $(0, L)$. Note that this is only possible since $f$ is strictly monotone.

Corollary 4.11 (Convergence rate on $(0, L)$ ). Let $u$ be the entropy solution of the initial-boundary value problem (4.8) on the bounded interval $[0, L]$ and $u_{\Delta t}$ the numerical approximation given by (4.9). Then we have the following convergence rate estimate:

$$
\left\|u(\cdot, T)-u_{\Delta t}(\cdot, T)\right\|_{\mathrm{L}^{1}(0, L)} \leq C \sqrt{\Delta x}
$$

for some constant $C$ independent of $\Delta x$.

Proof. Without repeating all calculations of Sections 4.1 and 4.2 we will highlight the adjustments to the respective proofs that need to be done. If we consider solutions on $(0, L)$ instead of $\mathbb{R}^{+}$the definition of $\Lambda_{\varepsilon, \varepsilon_{0}}(u, v)$ in 4.6 needs to be adjusted so that $\Lambda_{\varepsilon, \varepsilon_{0}}(u, v)$ contains the term

$$
-\int_{0}^{T} \int_{0}^{L} \int_{0}^{T} q(u(L-, t), v(y, s)) \varphi(L, t, y, s) \mathrm{d} t \mathrm{~d} y \mathrm{~d} s
$$

and all instances of $\mathbb{R}^{+}$need to be changed to $(0, L)$. Following the proofs of Theorems 4.4 and 4.10 in the same way finally yields

$$
\begin{array}{r}
\left\|u(\cdot, T)-u_{\Delta t}(\cdot, T)\right\|_{\mathrm{L}^{1}(0, L)} \\
+\int_{0}^{T} \int_{0}^{L} \int_{0}^{T}\left(q\left(u(L, t), u_{\Delta t}(y, s)\right)+q\left(u_{\Delta t}(L, t), u(y, s)\right)\right) \varphi(L, t, y, s) \mathrm{d} t \mathrm{~d} y \mathrm{~d} s \\
\leq C \sqrt{\Delta x}
\end{array}
$$


Using the monotonicity of $f$ we find

$$
q(u, v)=|f(u)-f(v)| \geq 0
$$

and thus the integral term in (4.13) is nonnegative which concludes the proof.

5. Statement and proof of the main theorem. Our main result now reads as follows:

THEOREM 5.1 (Convergence rate for conservation laws with discontinuous flux). Let $u$ be the entropy solution of Equation (1.1) and $u_{\Delta t}$ the numerical solution given by (3.1). Then we have the following convergence rate:

$$
\left\|u(\cdot, T)-u_{\Delta t}(\cdot, T)\right\|_{\mathrm{L}^{1}(\mathbb{R})} \leq C \sqrt{\Delta x}
$$

for some constant $C$ independent of $\Delta x$.

Proof. As before, we decompose the entropy solution $u$ as $u=\sum_{i=0}^{N} u^{(i)}$ where $u^{(i)}, i=0, \ldots, N$, are the respective entropy solutions on $D_{i}$, i.e., solutions of (2.3) and (2.4) respectively. Further, we decompose the numerical solution $u_{\Delta t}$ as $\sum_{i=0}^{N} u_{\Delta t}^{(i)}$ where

$$
u_{\Delta t}^{(i)}(x, t)= \begin{cases}u_{j}^{n} & \text { if }(x, t) \in \mathcal{C}_{j}^{n} \subset D_{i} \times \mathcal{C}^{n} \\ 0 & \text { otherwise }\end{cases}
$$

and $u_{j}^{n}$ is given by (3.1). Then we have

$$
\left\|u(T)-u_{\Delta t}(T)\right\|_{L^{1}(\mathbb{R})}=\sum_{i=0}^{N}\left\|u^{(i)}(T)-u_{\Delta t}^{(i)}(T)\right\|_{L^{1}\left(D_{i}\right)} .
$$

Using Theorem 4.4 for $D_{0}$, Theorem 4.10 for $D_{N}$, and Corollary 4.11 for each $D_{i}$, $i=1, \ldots, N-1$, shows that

$$
\left\|u^{(i)}(T)-u_{\Delta t}^{(i)}(T)\right\|_{\mathrm{L}^{1}\left(D_{i}\right)} \leq C \sqrt{\Delta x}
$$

for $i=0, \ldots, N$ which concludes the proof.

Remark 5.2. Note that the rate of Theorem 5.1 is optimal in the sense that it can not be improved without further assumptions on the initial datum. This can easily be shown in the same way as in the absence of spatial dependency since the specific initial datum $u_{0}$ constructed by Şabac in [34] can be chosen in a way such that $u_{0}$ is supported away from the last discontinuity.

6. Numerical experiments. To illustrate our results we now present two numerical experiments. We consider the 'two flux' case

$$
\begin{array}{cl}
u_{t}+(H(x) f(u)+(1-H(x)) g(u))_{x}=0, & (x, t) \in \mathbb{R} \times(0, T), \\
u(x, 0)=u_{0}(x), & x \in \mathbb{R}
\end{array}
$$

where $H$ is the Heaviside function. This corresponds to switching from one $u$ dependent flux, $g$, to another, $f$. 


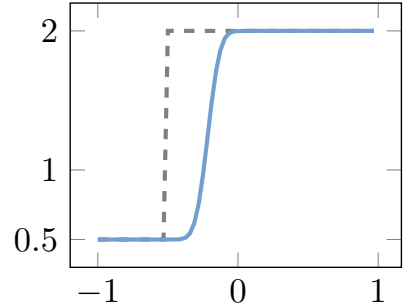

(a) $t=0.3$.

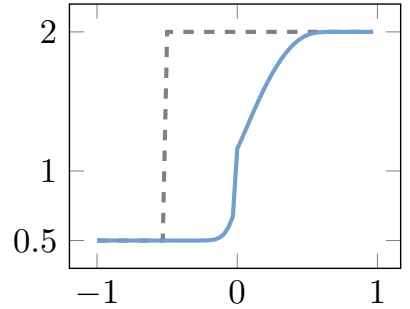

(b) $t=0.6$.

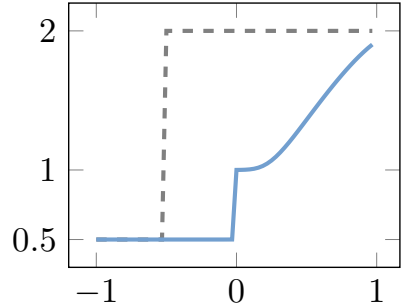

(c) $t=0.9$.

Fig. 1: Numerical solution of Experiment 1 with $\Delta x=2 / 64$ at various times.

Experiment 1. In our first numerical experiment we choose $g(u)=u$ and $f(u)=$ $\mathrm{u}^{2} / 2$ such that we switch from the transport equation to the Burgers equation across $x=0$. The initial datum we consider for Experiment 1 is

$$
u_{0}(x)= \begin{cases}0.5 & \text { if } x<-0.5 \\ 2 & \text { if } x>-0.5\end{cases}
$$

which is chosen such that the Rankine-Hugoniot condition at $x=0$ gives $u(0-, t)=$ $u(0+, t)$ before the jump at $x=-0.5$ interacts with the interface. Figure 1 shows the numerical solution calculated with the scheme (3.1) with open boundaries in blue and the initial datum in gray (dashed line) at various times (before, during, and after interaction with the interface). We used $\Delta x=2 / \mathrm{n}$ with $n=64$, end time $T=0.9$, and $\lambda=0.5$. We clearly recognize the characteristic features of the transport equation and the Burgers equation here as the upward jump in the initial datum is transported to the right as a shock until it crosses the interface at $x=0$ where the shock, as it enters the Burgers regime, subsequently becomes a rarefaction wave.

Experiment 2. In our second numerical experiment we choose $g(u)=\mathrm{u}^{2} / 2$ and $f(u)=u$ such that we switch from the Burgers equation to the transport equation across $x=0$. The initial datum we consider is

$$
u_{0}(x)=2+\exp \left(-100(x+0.75)^{2}\right) .
$$

Again, the offset of the initial datum is chosen in a way such that the RankineHugoniot condition at $x=0$ gives $u(0-, t)=u(0+, t)$ before the non-constant part of $u_{0}$ interacts with the interface. Figure 2 shows the numerical solution calculated with the scheme (3.1) with open boundaries in blue and the initial datum in gray (dashed line) at various times (immediately before, during, and after interaction with the interface). We used $\Delta x=2 / \mathrm{n}$ with $n=128$, end time $T=0.5$, and $\lambda=0.2$. We clearly recognize the shock formation due to the Burgers regime to the left of the interface (see Figure 2 (a)). Note that - although difficult to see in Figure 2 (c) because of numerical diffusion - the shock is preserved over the interface (only with a different profile).

Table 1 shows the observed convergence rates of the solution at time $T=0.9$ for Experiment 1 and at time $T=0.5$ for Experiment 2 for various values of $\Delta x$. As a reference solution, we used a numerical solution on a very fine grid $(n=2048)$ in both cases. As expected from experience in the case of spatially independent flux we observe convergence rates strictly between $1 / 2$ and 1 (cf. e.g. [24, clawpack software]). 


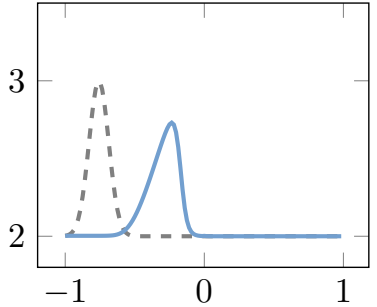

(a) $t=0.2$.

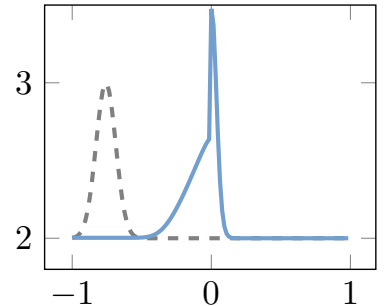

(b) $t=0.3$.

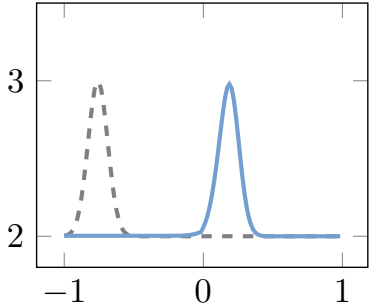

(c) $t=0.5$.

Fig. 2: Numerical solution of Experiment 2 with $\Delta x=2 / 128$ at various times.

\begin{tabular}{rcc}
\hline \multicolumn{1}{c}{$n$} & $\mathrm{~L}^{1}$ error & $\mathrm{L}^{1}$ OOC \\
\hline 16 & $1.751 \times 10^{-1}$ & - \\
32 & $1.256 \times 10^{-1}$ & 0.48 \\
64 & $8.865 \times 10^{-2}$ & 0.50 \\
128 & $5.918 \times 10^{-2}$ & 0.58 \\
256 & $3.637 \times 10^{-2}$ & 0.70 \\
512 & $1.978 \times 10^{-2}$ & 0.88 \\
1024 & $8.145 \times 10^{-3}$ & 1.28 \\
\hline
\end{tabular}

(a) Experiment 1.

\begin{tabular}{rcc}
\hline \multicolumn{1}{c}{$n$} & $\mathrm{~L}^{1}$ error & $\mathrm{L}^{1}$ OOC \\
\hline 16 & $2.771 \times 10^{-1}$ & - \\
32 & $1.823 \times 10^{-1}$ & 0.60 \\
64 & $1.261 \times 10^{-1}$ & 0.53 \\
128 & $8.390 \times 10^{-2}$ & 0.59 \\
256 & $5.125 \times 10^{-2}$ & 0.71 \\
512 & $2.780 \times 10^{-2}$ & 0.88 \\
1024 & $1.132 \times 10^{-2}$ & 1.30 \\
\hline
\end{tabular}

(b) Experiment 2.

Table 1: Convergence rates of Experiment 1 and 2.

7. Conclusion. Scalar conservation laws with discontinuous flux frequently occur in physical applications and several numerical schemes have been considered in the literature. In contrast to the case where the nonlinear flux does not have a spatial dependency, however, convergence rate results for monotone finite volume schemes have not been available until now.

In this paper, we have established a convergence rate for upwind-type finite volume methods for the case where $f$ is strictly monotone in $u$ and the spatial dependency $k$ is piecewise constant with finitely many discontinuities. The central idea of this paper is to split the problem into finitely many conservation laws between two neighboring discontinuities of $k$ and thus get a convergence rate as a consequence of convergence rates on bounded domains. Here, the novel feature of this paper is the strong bound on the temporal total variation of the finite volume approximation which allows us to estimate the boundary terms in space at the discontinuities of $k$ that appear when applying the classical Kuznetsov theory to problem (1.1).

As an outlook we name four possible directions of future research. A first direction would be to extend the convergence rate result of this paper to the cases where $k$ is not piecewise constant and $f$ is not monotone. Second, it might be interesting to investigate convergence rates of monotone schemes in the Wasserstein distance. In the case of spatially independent fluxes, convergence rates in the Wasserstein distance are well-understood due to Nessyahu, Tadmor and Tassa [28, 29]. A third direction of future research might be to see whether the results of this paper can be extended to monotone schemes in conservation form, i.e., where the definition of $u_{P_{i}}^{n+1}$ in (3.1) 
is replaced by $u_{P_{i}}^{n+1}=u_{P_{i}}^{n}-\lambda\left(f^{(i)}\left(u_{P_{i}}^{n}\right)-f^{(i-1)}\left(u_{P_{i}-1}^{n}\right)\right)$. Lastly, convergence rates of the front tracking method for conservation laws with discontinuous flux are highly desirable as well. In the case of spatially independent fluxes, convergence rates of the front tracking method are known in $\mathrm{L}^{1}$ due to Lucier [26] and in the Wasserstein distances due to Solem [35].

Acknowledgments. We thank Nils Henrik Risebro for several useful discussions and Ulrik Skre Fjordholm for his careful reading of the manuscript. We also like to thank the referees for their constructive and insightful comments.

Appendix A. Convergence rate estimates for general initial-boundary value problems. With the techniques developed in this paper, we can also derive a convergence rate for the initial-boundary value problem

$$
\begin{array}{ll}
u_{t}+f(u)_{x}=0, & (x, t) \in(0, L) \times(0, T), \\
u(x, 0)=u_{0}(x), & x \in(0, L), \\
u(0, t)=a(t), & t \in(0, T)
\end{array}
$$

and the numerical scheme

$$
\begin{gathered}
u_{j}^{n+1}=u_{j}^{n}-\lambda\left(f\left(u_{j}^{n}\right)-f\left(u_{j-1}^{n}\right)\right), \quad j \geq 1, n \geq 0 \\
u_{j}^{0}=\frac{1}{\Delta x} \int_{\mathcal{C}_{j}} u_{0}(x) \mathrm{d} x, \quad j \geq 0, \\
u_{0}^{n}=\frac{1}{\Delta t} \int_{\mathcal{C}^{n}} a(s) \mathrm{d} s, \quad n \geq 1 .
\end{gathered}
$$

Here we need to assume that $a \in\left(\mathrm{L}^{1} \cap \mathrm{BV}\right)(0, T)$ which allows us to use the total variation of $a$ directly instead of crossing the discontinuity in Lemma 4.6. The assertion of Lemma 4.6 should then read

$$
\sum_{n=0}^{M}\left|u_{j}^{n+1}-u_{j}^{n}\right| \leq C\left(\operatorname{TV}\left(u_{0}\right)+\operatorname{TV}(a)\right)
$$

which can be used at the same place Lemma 4.6 is used in Theorem 4.10. Hence, Corollary 4.11 gives the convergence rate $\mathcal{O}(\sqrt{\Delta x})$ for the general initial-boundary value problem (A.1). Note that this is a higher rate than the $\mathcal{O}\left(\Delta x^{1 / 3}\right)$ rate mentioned in [30].

\section{REFERENCES}

[1] Adimurthi, S. Mishra, And G. V. Gowda, Conservation law with the flux function discontinuous in the space variable-II: Convex-concave type fluxes and generalized entropy solutions, Journal of Computational and Applied Mathematics, 203 (2007), pp. $310-344$.

[2] Adimurthi, S. Misra, And G. V. Gowda, Optimal entropy solutions for conservation laws with discontinuous flux-functions, Journal of Hyperbolic Differential Equations, 2 (2005), pp. $783-837$.

[3] B. Andreianov, K. H. Karlsen, and N. H. Risebro, A theory of $L^{1}$-dissipative solvers for scalar conservation laws with discontinuous flux, Archive for rational mechanics and analysis, 201 (2011), pp. 27-86.

[4] E. Audusse and B. Perthame, Uniqueness for scalar conservation laws with discontinuous flux via adapted entropies, Proceedings of the Royal Society of Edinburgh Section A: Mathematics, 135 (2005), pp. 253-265.

[5] J. Badwaik, N. H. Risebro, And C. Klingenberg, Multilevel Monte Carlo finite volume methods for random conservation laws with discontinuous flux, arXiv preprint arXiv:1906.08991, (2019). 
[6] P. Baiti And H. K. Jenssen, Well-posedness for a class of $2 \times 2$ conservation laws with $L^{\infty}$ data, Journal of Differential Equations, 140 (1997), pp. $161-185$.

[7] R. Bürger, K. Karlsen, C. Klingenberg, and N. Risebro, A front tracking approach to a model of continuous sedimentation in ideal clarifier-thickener units, Nonlinear Analysis: Real World Applications, 4 (2003), pp. 457-481.

[8] G. Coclite, J. Ridder, AND N. Risebro, A convergent finite difference scheme for the Ostrovsky-Hunter equation on a bounded domain, BIT Numerical Mathematics, 57 (2017), pp. 93-122.

[9] G. M. Coclite And N. H. Risebro, Conservation laws with time dependent discontinuous coefficients, SIAM Journal on Mathematical Analysis, 36 (2005), pp. 1293-1309.

[10] M. G. Crandall and A. Majda, Monotone difference approximations for scalar conservation laws, Mathematics of Computation, 34 (1980), pp. 1-21.

[11] S. DieHL, A conservation law with point source and discontinuous flux function modelling continuous sedimentation, SIAM Journal on Applied Mathematics, 56 (1996), pp. 388419.

[12] T. Gimse And N. H. Risebro, Riemann problems with a discontinuous flux function, in Proceedings of Third International Conference on Hyperbolic Problems, vol. 1, 1991, pp. 488502.

[13] T. Gimse And N. H. Risebro, Solution of the cauchy problem for a conservation law with a discontinuous flux function, SIAM Journal on Mathematical Analysis, 23 (1992), pp. 635648.

[14] J. Greenberg, A. Leroux, R. Baraille, And A. Noussair, Analysis and approximation of conservation laws with source terms, SIAM Journal on Numerical Analysis, 34 (1997), pp. 1980-2007.

[15] H. Holden And N. H. Risebro, Front tracking for hyperbolic conservation laws, vol. 152, Springer, 2015.

[16] K. Karlsen, N. Risebro, And J. Towers, Upwind difference approximations for degenerate parabolic convection-diffusion equations with a discontinuous coefficient, IMA Journal of Numerical Analysis, 22 (2002), pp. 623-664.

[17] K. H. Karlsen, N. H. Risebro, and J. D. Towers, On a nonlinear degenerate parabolic transport-diffusion equation with a discontinuous coefficient., Electronic Journal of Differential Equations, (2002), p. (electronic).

[18] K. H. Karlsen and J. D. Towers, Convergence of the Lax-Friedrichs scheme and stability for conservation laws with a discontinuous space-time dependent flux, Chinese Annals of Mathematics, 25 (2004), pp. 287-318.

[19] R. A. Klausen and N. H. Risebro, Stability of conservation laws with discontinuous coeffcients, Journal of Differential Equations, 157 (1999), pp. 41-60.

[20] C. Klingenberg And N. H. Risebro, Convex conservation laws with discontinuous coeffcients. Existence, uniqueness and asymptotic behavior, Communications in Partial Differential Equations, 20 (1995), pp. 1959-1990.

[21] C. Klingenberg And N. H. Risebro, Stability of a resonant system of conservation laws modeling polymer flow with gravitation, Journal of Differential Equations, 170 (2001), pp. 344380.

[22] S. N. KRUŽKov, First order quasilinear equations in several independent variables, Mathematics of the USSR-Sbornik, 10 (1970), pp. 217-243.

[23] N. Kuznetsov, Accuracy of some approximate methods for computing the weak solutions of a first-order quasi-linear equation, USSR Computational Mathematics and Mathematical Physics, 16 (1976), pp. 105-119.

[24] R. J. LeVeque, Finite volume methods for hyperbolic problems, Cambridge Texts in Applied Mathematics, Cambridge University Press, Cambridge, 2002.

[25] M. J. Lighthill and G. B. Whitham, On kinematic waves II. A theory of traffic flow on long crowded roads, Proceedings of the Royal Society of London. Series A. Mathematical and Physical Sciences, 229 (1955), pp. 317-345.

[26] B. J. LuCIER, A moving mesh numerical method for hyperbolic conservation laws, Mathematics of Computation, 46 (1986), pp. 59-69.

[27] S. Mishra, Convergence of upwind finite difference schemes for a scalar conservation law with indefinite discontinuities in the flux function, SIAM Journal on Numerical Analysis, 43 (2005), pp. 559-577.

[28] H. Nessyahu ANd E. TADMOR, The convergence rate of approximate solutions for nonlinear scalar conservation laws, SIAM Journal on Numerical Analysis, 29 (1992), pp. 1505-1519.

[29] H. Nessyahu, E. Tadmor, and T. Tassa, The convergence rate of Godunov type schemes, SIAM Journal on Numerical Analysis, 31 (1994), pp. 1-16. 
[30] M. Ohlberger AND J. Vovelle, Error estimate for the approximation of nonlinear conservation laws on bounded domains by the finite volume method, Mathematics of Computation, 75 (2006), pp. 113-150.

[31] J. RidDER AND A. M. RuF, A convergent finite difference scheme for the Ostrovsky-Hunter equation with Dirichlet boundary conditions, BIT Numerical Mathematics, (2019).

[32] N. H. Risebro AND A. TVeito, Front tracking applied to a nonstrictly hyperbolic system of conservation laws, SIAM Journal on Scientific and Statistical Computing, 12 (1991), pp. 1401-1419.

[33] A. M. Ruf, E. Sande, And S. Solem, The optimal convergence rate of monotone schemes for conservation laws in the Wasserstein distance, Journal of Scientific Computing, (2019).

[34] F. ŞABAC, The optimal convergence rate of monotone finite difference methods for hyperbolic conservation laws, SIAM Journal on Numerical Analysis, 34 (1997), pp. 2306-2318.

[35] S. Solem, Convergence rates of the front tracking method for conservation laws in the Wasserstein distances, SIAM Journal on Numerical Analysis, 56 (2018), pp. 3648-3666.

[36] Z.-H. Teng And P. Zhang, Optimal $L^{1}$-rate of convergence for the viscosity method and monotone scheme to piecewise constant solutions with shocks, SIAM Journal on Numerical Analysis, 34 (1997), pp. 959-978.

[37] J. Towers, Convergence of a difference scheme for conservation laws with a discontinuous flux, SIAM Journal on Numerical Analysis, 38 (2000), pp. 681-698.

[38] J. Towers, A difference scheme for conservation laws with a discontinuous flux: The nonconvex case, SIAM Journal on Numerical Analysis, 39 (2001), pp. 1197-1218.

[39] D. A. Venditti And D. L. Darmofal, Adjoint error estimation and grid adaptation for functional outputs: Application to quasi-one-dimensional flow, Journal of Computational Physics, 164 (2000), pp. 204-227.

[40] X. WEN AND S. Jin, Convergence of an immersed interface upwind scheme for linear advection equations with piecewise constant coefficients I: $L^{1}$-error estimates, Journal of Computational Mathematics, (2008), pp. 1-22. 